I.V. STASYUK, O.V. VELYCHKO, I.R. DULEPA

Institute for Condensed Matter Physics, Nat. Acad. of Sci. of Ukraine

(1, Svientsitskii Str., Lviv 79011, Ukraine)

\title{
INVESTIGATION OF THE BOSONIC SPECTRUM OF TWO-DIMENSIONAL OPTICAL GRAPHENE-TYPE LATTICES. SUPERFLUID PHASE
} PACS 37.10.Jk, 67.85.-d

\begin{abstract}
The energy spectrum of a system of Bose atoms in the superfluid phase in an optical lattice of the graphene type has been studied. The dispersion laws for the energy bands and the single particle spectral densities are calculated in the random phase approximation and in the framework of the hard-core boson formalism, and their changes at the transition from the normal phase to the superfluid one are described. As a result of this transformation, the number of subbands doubles. In the case of the subband energetic equivalence, the Dirac points in the spectrum survive, and their number becomes twice as much. When the subbands are energetically nonequivalent, the Dirac points are absent. The shape of spectral densities is shown to be sensitive to the changes in the temperature and the chemical potential position.

Ke ywords: optical lattice, honeycomb lattice, phase transition, spectral density, hard-core bosons, Dirac points.
\end{abstract}

\section{Introduction}

This work continues our researches dealing with the calculation of the energy spectrum and one-particle spectral densities for a system of Bose atoms in a two-dimensional honeycomb optical lattice of the graphene type. Our previous results were reported in work [1]. Unlike the problem of the electron spectrum in graphene, we consider particles that are described by the non-Fermi statistics. During the last time, a considerable attention was attracted to the study of the thermodynamics and the energy spectrum features of Bose particles in optical lattices. Interesting and important is the problem concerning the spectrum modifications at the phase transition associated with the Bose-Einstein condensation of particles (the transition from the normal, NO, phase to the superfluid, SF, one).

The Bose-Einstein (BE) condensation of bosonic atoms $\left(\mathrm{Rb}^{87}\right)$ in the optical lattices formed as a result of the interference between counter-propagating laser beams was observed for the first time in 2002

(C) I.V. STASYUK, O.V. VELYCHKO, I.R. DULEPA, 2014 ISSN 2071-0194. Ukr. J. Phys. 2014. Vol. 59, No. 10
$[2,3]$. Since that time, this effect and the accompanying phenomena have been studied very intensively. For optical lattices of the graphene type, the transition to the SF phase was obtained experimentally in work [4], where the regions of existence for various phases depending on the values of chemical potential and parameters that characterize shortrange interactions and the particle dynamics were found. The theoretical consideration in works [5-8] concerned the construction and the analysis of phase diagrams, proceeding from the Bose-Hubbard model $[9,10]$, which is generally adopted for the description of a system of Bose atoms in optical lattices. The energy spectrum of bosons in the graphene-type lattices was analyzed in works $[11,12]$. Issues concerning the spectrum topology and the arrangement of Dirac points in the NO phase were considered, but the spectrum modifications at the transition to the SF phase were not analyzed.

An important influence on the formation of a spectrum of the system of Bose atoms in the graphenetype lattice is exerted by short-range correlations between particles, (in particular, the on-site repulsion 
interaction $U$ ). Another complication in comparison with the case of the graphene lattice is the energetic nonequivalence of sublattices (it can be easily controlled by changing the phases of laser beams that generate the optical lattice). As was shown in work [1], all those factors give rise to substantial differences between the single particle spectrum in the NO phase and the standard spectrum of graphene. In particular, it was found that Dirac points in the spectrum do not exist at arbitrary particle densities and, accordingly, chemical potential values. In particular, they disappear, if the chemical potential in the NO phase is located in the gap between the energy subbands (such a gap emerges owing to the energetic nonequivalence of sublattices).

In this work, in addition to calculations carried out in work [1], we consider the case where the system is in the phase with the BE condensate (the SF phase). Our description is based, as was done in work [1], on the two-sublattice model of hard-core bosons [13], which is a limiting case $(U \rightarrow \infty)$ of the BoseHubbard model and is valid for the low-population levels $(0 \leq n \leq 1)$ of on-site states. The general scheme to find Green's single particle functions for this model in its pseudo-spin formulation is wellknown. In this work, we use the approach described in work [14]. We aim at studying the special features in the reconstruction of the bosonic band spectrum and spectral densities at the transition from the NO phase to the SF one that occurs at the variations of the chemical potential, energetic difference between the sublattices, and temperature.

\section{Excitation}

\section{Spectrum in the Superfluid Phase}

If the intersite interaction between particles is neglected, the Hamiltonian of the lattice gas consisted of hard-core bosons looks like

$H=-\sum_{\langle i j\rangle} t b_{i}^{+} b_{j}+\sum_{i}\left(\varepsilon_{0}-\mu\right) n_{i}$,

where $t$ is the transfer integral between the nearest sites, $\varepsilon_{0}$ the on-site energy of the particle, and $\mu$ the chemical potential. Since $b_{i}^{+}$and $b_{i}$ are the Pauli operators, Hamiltonian (1) can be rewritten in the pseudo-spin representation with the use of the transformations

$b_{i}^{+}=S_{i}^{-}, \quad b_{i}=S_{i}^{+}, \quad b_{i}^{+} b_{i}=n_{i}=\frac{1}{2}-S_{i}^{z}$.
In the two-sublattice case, $i \rightarrow(n, \alpha)$, where $\alpha=A, B$ is the sublattice index. As a result,

$$
\begin{aligned}
& \hat{H}=-\sum_{\left\langle n, n^{\prime}\right\rangle}\left[J _ { n n ^ { \prime } } ^ { A B } \left(S_{n A}^{x} S_{n^{\prime} B}^{x}+\right.\right. \\
& \left.+J_{n n^{\prime}}^{B A}\left(S_{n B}^{x} S_{n^{\prime} A}^{x}+S_{n B}^{y} S_{n^{\prime} A}^{y}\right)\right]- \\
& -h_{A} \sum_{n} S_{n A}^{z}-h_{B} \sum_{n} S_{n B}^{z} \\
& \left(J_{\left\langle n, n^{\prime}\right\rangle}^{A B}=J_{\left\langle n^{\prime}, n\right\rangle}^{B A}=t, \quad h_{\alpha}=\varepsilon_{\alpha}-\mu\right) .
\end{aligned}
$$

In the phase with a Bose condensate, the order parameter is the non-zero average

$$
\begin{aligned}
& S_{n \alpha}^{z}=\sigma_{n \alpha}^{z} \cos \vartheta_{\alpha}+\sigma_{n \alpha}^{x} \sin \vartheta_{\alpha}, \\
& S_{n \alpha}^{x}=\sigma_{n \alpha}^{x} \cos \vartheta_{\alpha}-\sigma_{n \alpha}^{z} \sin \vartheta_{\alpha}, \\
& S_{n \alpha}^{y}=\sigma_{n \alpha}^{y},
\end{aligned}
$$

where the angles $\vartheta_{\alpha}$ are determined by diagonalizing the mean-field Hamiltonian

$H_{\mathrm{MF}}=-\sum_{n \alpha} E_{\alpha} \sigma_{n \alpha}^{z}$.

Since $\left\langle\sigma_{n \alpha}^{z}\right\rangle \neq 0$ and $\left\langle\sigma_{n \alpha}^{x}\right\rangle=\left\langle\sigma_{n \alpha}^{y}\right\rangle=0$, we obtain $\left\langle S_{\alpha}^{x}\right\rangle=-\left\langle\sigma_{\alpha}^{z}\right\rangle \sin \vartheta_{\alpha}$.

In the normal phase,

$\sin \vartheta_{\alpha}=0, \quad E_{\alpha}=h_{\alpha}, \quad\left\langle\sigma_{n \alpha}^{z}\right\rangle=\frac{1}{2} \tanh \frac{\beta h_{\alpha}}{2}$.

For the SF phase, the internal fields $E_{\alpha}$ and angles $\vartheta_{\alpha}$ can be determined from the system of equations [14]

$$
\begin{aligned}
& \sin ^{2} \vartheta_{\alpha}=\frac{\left\langle\sigma_{\alpha}^{z}\right\rangle^{2}\left\langle\sigma_{\beta}^{z}\right\rangle^{2} J^{4}(0)-h_{\alpha}^{2} h_{\beta}^{2}}{\left\langle\sigma_{\alpha}^{z}\right\rangle^{2} J^{2}(0)\left[h_{\alpha}^{2}+\left\langle\sigma_{\beta}^{z}\right\rangle^{2} J^{2}(0)\right]}, \\
& \left\langle\sigma_{\alpha}^{z}\right\rangle=\frac{1}{2} \tanh \frac{\beta E_{\alpha}}{2} \\
& E_{\alpha}=\left\langle\sigma_{\alpha}^{z}\right\rangle J(0) \frac{\sqrt{h_{\alpha}^{2}+\left\langle\sigma_{\beta}^{z}\right\rangle^{2} J^{2}(0)}}{\sqrt{h_{\beta}^{2}+\left\langle\sigma_{\alpha}^{z}\right\rangle^{2} J^{2}(0)}}
\end{aligned}
$$

and the transition from one phase into another one occurs when the condition

$h_{A} h_{B}=\left\langle\sigma_{A}^{z}\right\rangle\left\langle\sigma_{B}^{z}\right\rangle J^{2}(0) \equiv\left\langle\sigma_{A}^{z}\right\rangle\left\langle\sigma_{B}^{z}\right\rangle 9 t^{2}$

is satisfied. Relation (8) determines the boundaries of regions for the NO and SF phases on the phase

ISSN 2071-0194. Ukr. J. Phys. 2014. Vol. 59, No. 10 
plane. In Fig. 1, the corresponding phase diagrams on the plane $(T, h)[1,14]$, where $h=\frac{h_{A}+h_{B}}{2}$, are exhibited for various values of parameter $\delta=\frac{h_{A}-h_{B}}{2}$.

Green's two-time function

$\left\langle\left\langle b_{l \alpha} \mid b_{n \beta}^{+}\right\rangle\right\rangle=\left\langle\left\langle S_{l \alpha}^{+} \mid S_{n \beta}^{-}\right\rangle\right\rangle \equiv G_{l \alpha, n \beta}^{+-}$,

which can be used to obtain the bosonic spectrum, was found in works $[1,14]$, by using the method of equations of motion in the random phase approximation. In the momentum-frequency representation, its Fourier transform looks like

$$
\begin{aligned}
& \left\langle\left\langle S_{\mathrm{A}}^{+} \mid S_{\mathrm{A}}^{-}\right\rangle\right\rangle_{q, w}=\frac{\hbar}{2 \pi}\left\langle\sigma_{\mathrm{A}}^{z}\right\rangle P_{q}^{\mathrm{A}}\left[\left(\hbar^{2} \omega^{2}-E_{\mathrm{A}}^{2}\right)\left(\hbar^{2} \omega^{2}-E_{\mathrm{B}}^{2}\right)-\right. \\
& \left.-2 M_{q} \hbar^{2} \omega^{2}-2 N_{q} E_{\mathrm{A}} E_{\mathrm{B}}+M_{q}^{2}\right]^{-1}
\end{aligned}
$$

where the numerator of Green's function equals

$$
\begin{aligned}
& P_{q}^{\mathrm{A}}(\hbar \omega)=\left[E_{\mathrm{A}}\left(\cos ^{2} \vartheta_{\mathrm{A}}+1\right)+2 \hbar \omega \cos \vartheta_{\mathrm{A}}\right] \times \\
& \times\left(\hbar^{2} \omega^{2}-E_{\mathrm{B}}^{2}\right)-2 \hbar \omega M_{q} \cos \vartheta_{\mathrm{A}}+\tilde{\Phi}_{q}^{\mathrm{A}} E_{\mathrm{B}}
\end{aligned}
$$

and the following notations are introduced:

$M_{q}=\Phi_{q} \cos \vartheta_{\mathrm{A}} \cos \vartheta_{\mathrm{B}}$,

$N_{q}=\frac{1}{2} \Phi_{q}\left(1+\cos ^{2} \vartheta_{\mathrm{A}} \cos ^{2} \vartheta_{\mathrm{B}}\right)$,

$\tilde{\Phi}_{q}^{\mathrm{A}}=\Phi_{q} \cos ^{2} \vartheta_{\mathrm{A}}\left(1+\cos ^{2} \vartheta_{\mathrm{B}}\right)$,

$\Phi_{q}=\left\langle\sigma_{A}^{z}\right\rangle\left\langle\sigma_{B}^{z}\right\rangle J^{2}(q)$.

Here,

$$
\begin{aligned}
& J(\mathbf{q})=t\left[\mathrm{e}^{\mathrm{i} q_{y} a}+2 \mathrm{e}^{-\mathrm{i} q_{y} a} \cos \left(q_{x} \frac{\sqrt{3} a}{2}\right)\right], \\
& J(0)=3 t
\end{aligned}
$$

and $a$ is the distance between the nearest neighbors in the honeycomb lattice.

The excitation spectrum of bosons in the SF phase consists of four, symmetric in pairs, branches,

$$
\begin{aligned}
& \varepsilon_{1,2}^{(\mathrm{SF})}(\mathbf{q})= \pm\left(P_{q}+Q_{q}\right)^{1 / 2}, \\
& \varepsilon_{3,4}^{(\mathrm{SF})}(\mathbf{q})= \pm\left(P_{q}-Q_{q}\right)^{1 / 2},
\end{aligned}
$$

where

$$
\begin{aligned}
& P_{q}=\frac{1}{2}\left(E_{\mathrm{A}}^{2}+E_{\mathrm{B}}^{2}\right)+M_{q}, \\
& Q_{q}=\left[\frac{1}{4}\left(E_{\mathrm{A}}^{2}-E_{\mathrm{B}}^{2}\right)^{2}+2 N_{q} E_{\mathrm{A}} E_{\mathrm{B}}+\right. \\
& \left.+M_{q}\left(E_{\mathrm{A}}^{2}+E_{\mathrm{B}}^{2}\right)\right]^{1 / 2} .
\end{aligned}
$$

ISSN 2071-0194. Ukr. J. Phys. 2014. Vol. 59, No. 10

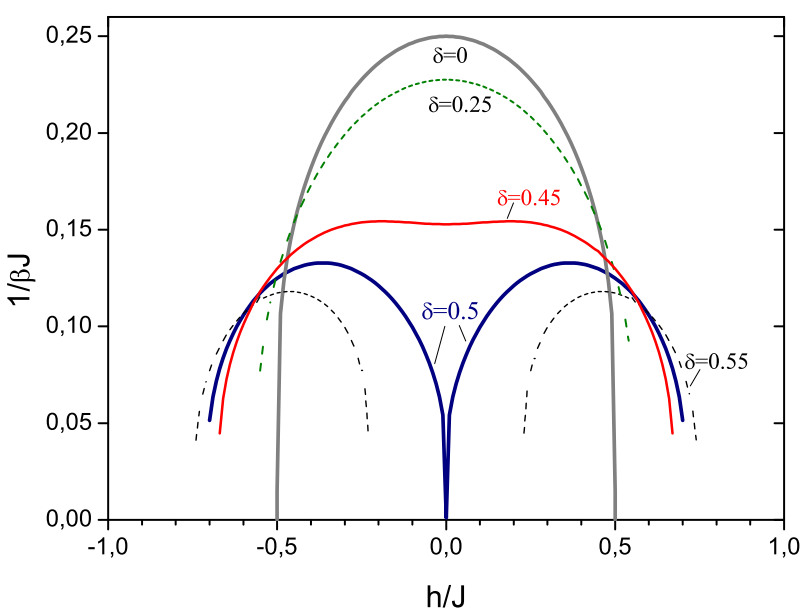

Fig. 1. Phase diagrams in the $(T, h)$ plane at $\delta=0,0.25$, $0.45,0.5$, and $0.55[14]$

In comparison with the NO phase, where [14]

$\varepsilon_{1,2}(\mathbf{q})=h \pm \sqrt{\delta^{2}+\left\langle\sigma_{\mathrm{A}}^{z}\right\rangle\left\langle\sigma_{\mathrm{B}}^{z}\right\rangle|J(\mathbf{q})|^{2}}$,

the number of branches is twice as much. The duplication of the specular reflection type with respect to the chemical potential level takes place at the phase transition, when the variation of model parameters $(\mu, \delta$, or $T)$ gives rise to the situation where the edge of either subband (16) touches the $\mu$-level. This situation is illustrated in Figs. 2 and 3 in the cases where the chemical potential is initially (in the NO phase) located between the subbands or under them. In the latter case when the gap in the NO phase spectrum is absent, i.e. at $\delta=0$, there are the Dirac points with a linear dispersion law on the Brillouin zone boundary. They are an analog of such points in the electron band spectrum of graphene (see, e.g., work [15]). The number of Dirac points doubles after the transition into the SF phase. New points of this type appear in the region of negative energies, if the bosonic band in the NO phase is located above the chemical potential level, or in the region of positive energies, if below it (see Fig. 3).

Note that, if the chemical potential $\mu$ is located between the subbands (in this case, it must be $\delta \neq 0$ ), the spectrum is reconstructed, and the Dirac points disappear. The situation where the chemical potential is located in a close vicinity of the Dirac points or is imposed onto them is not realized in the case of hard-core bosons (except for the regions near the 

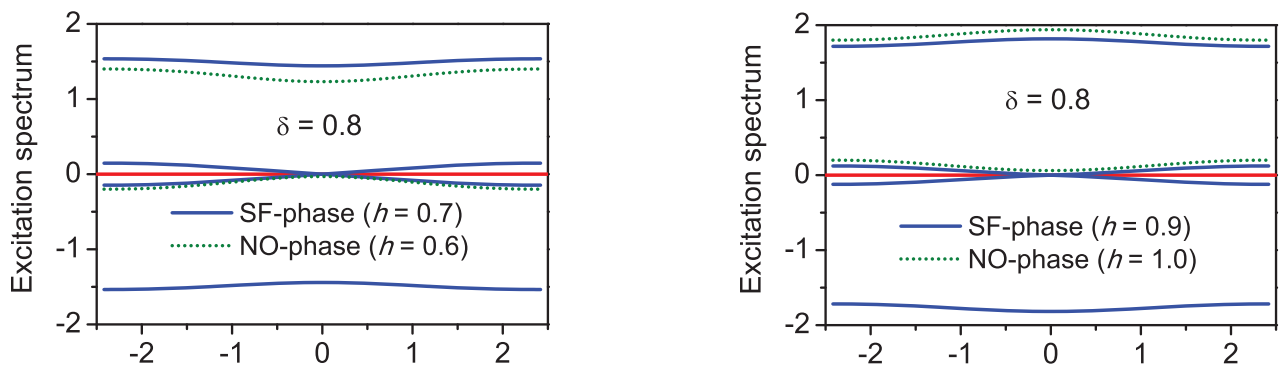

Fig. 2. Forms of spectral branches and their correspondence to the NO (dotted curves) and SF (solid curves) phases in the cases where the chemical potential in the NO phase is located between the bands (left panel; $h=0.6$ in the NO phase and 0.7 in the SF one) and under them (right panel; $h=0.9$ in the SF phase and 1.0 in the NO one). The other parameters are $\delta=0.8$ and $\Theta=0.05$. Hereafter, the energy is reckoned in the $J(0)$-units from the chemical potential
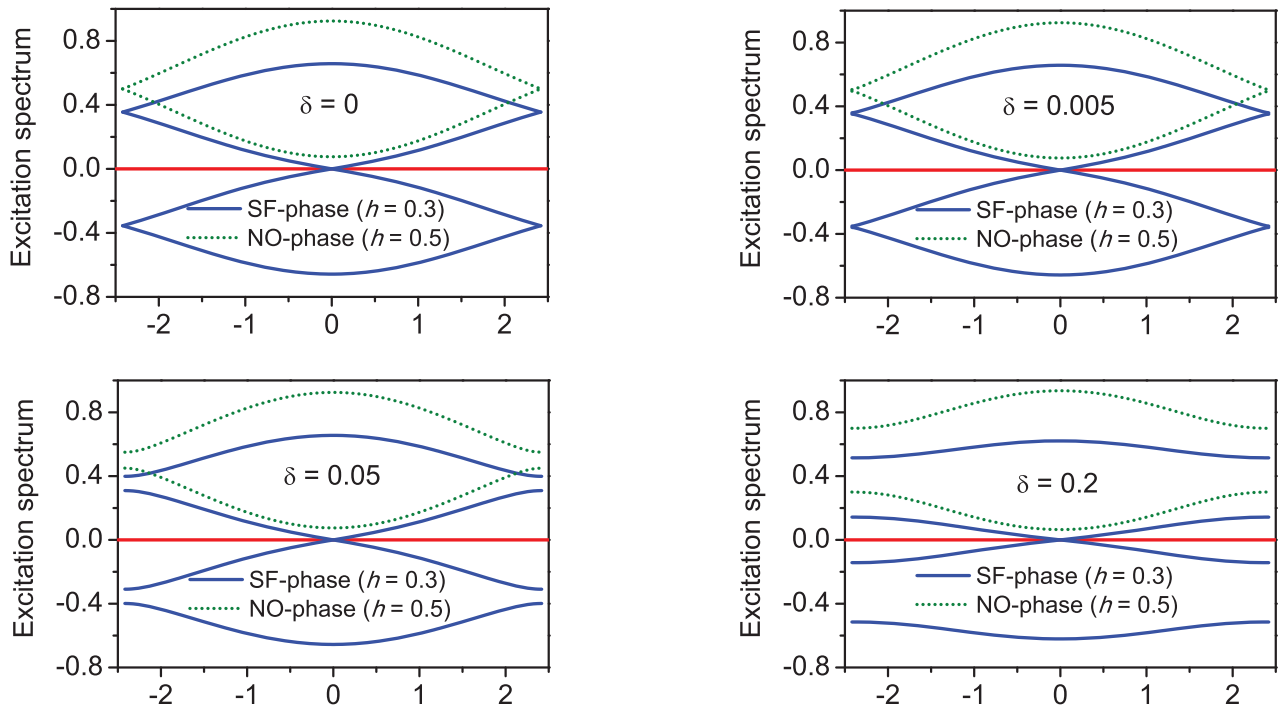

Fig. 3. Appearance of new Dirac points at $\delta=0$, the divergency of branches at the Brillouin zone boundaries as the $\delta$ parameter grows, and a change in the behaviour of branches at the center of the Brillouin zone at the NO $\rightarrow \mathrm{SF}$ transition $(h=0.3$ in the $\mathrm{SF}$ phase and 0.5 in the $\mathrm{NO}$ one; $\Theta=0.2$ )

critical points, which are the maximum points for the curves of phase equilibrium on the phase diagram in Fig. 1).

In the SF phase, two central subbands always touch each other at the point $\mathbf{q}=0$ at the chemical potential level. In a vicinity of this point, the dispersion laws in the subbands are linear, and, in this sense, they correspond to excitations of the Bogolyubov type in the interacting Bose gas, which exist in the presence of a BE condensate [16].

\section{Spectral}

\section{Density $\rho_{\alpha}(\omega)$ in the SF Phase}

The spectral density function for single particle bosonic excitations calculated per one lattice site will be designated here as

$$
\begin{aligned}
& \rho_{\alpha}(\omega)=-\frac{2}{N} \sum_{\mathbf{q}} \operatorname{Im}\left\langle\left\langle b_{\alpha} \mid b_{\alpha}^{+}\right\rangle\right\rangle_{\mathbf{q}, \omega+\mathrm{i} \varepsilon}= \\
& =-\frac{2}{N} \sum_{\mathbf{q}} \operatorname{Im}\left\langle\left\langle S_{\alpha}^{+} \mid S_{\alpha}^{-}\right\rangle\right\rangle_{\mathbf{q}, \omega+\mathrm{i} \varepsilon} .
\end{aligned}
$$

Proceeding from formula (10) and making a decomposition into simple fractions, the function $\rho_{\alpha}(\omega)$ can be written as follows:

$$
\rho_{\alpha}(\omega)=\frac{1}{N} \sum_{q}\left\langle\sigma_{\alpha}^{z}\right\rangle \sum_{i=1}^{4} A_{i}^{\alpha}(\mathbf{q}) \delta\left(\omega-\frac{\varepsilon_{i}(\mathbf{q})}{\hbar}\right)
$$

ISSN 2071-0194. Ukr. J. Phys. 2014. Vol. 59, No. 10 

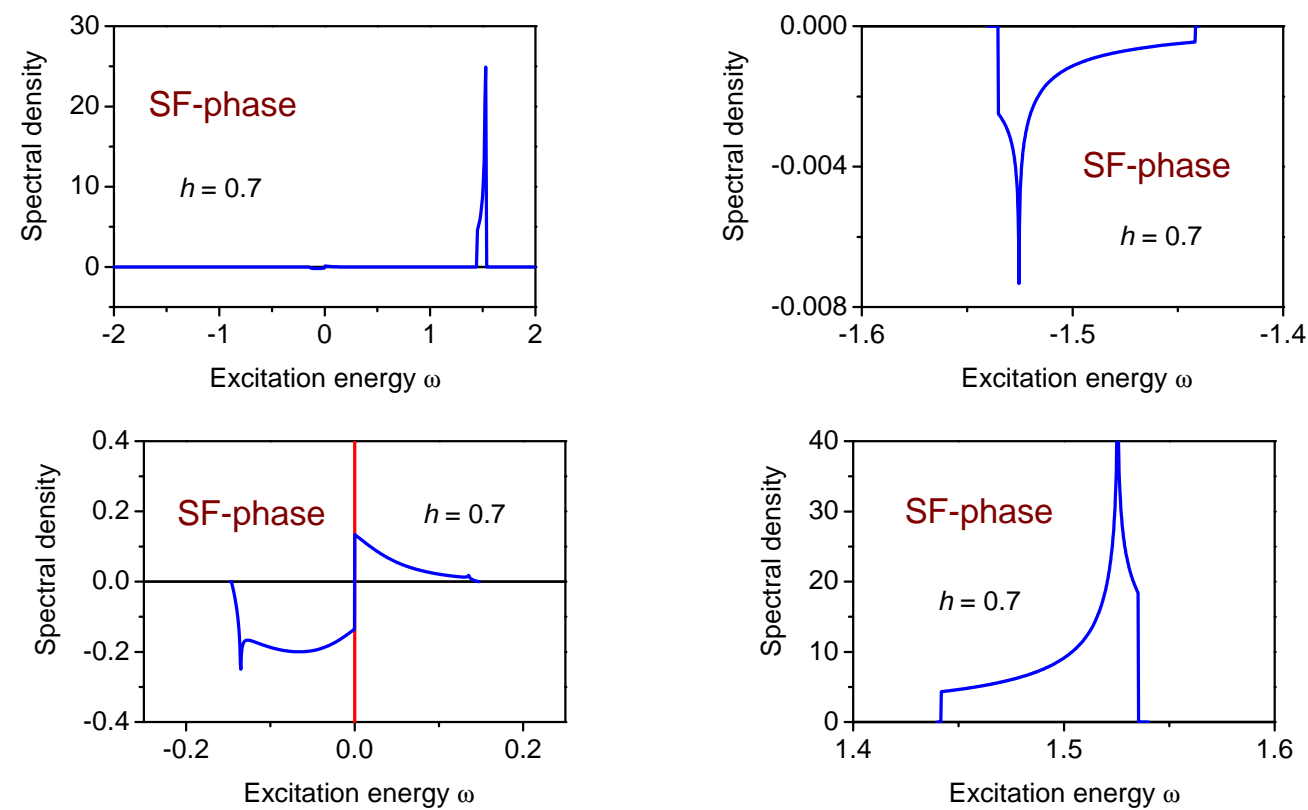

Fig. 4. Spectral density $\rho_{A}(\omega)$ in the SF phase: general view (upper left panel) and scaled-up images for three intervals of the excitation energy $\hbar \omega$, where the spectral density differs from zero (other panels; the weights of different subbands differ by orders of magnitude). The parameter values $J(0)=1, \delta=0.8, h=0.7$, and $\Theta=0.05$

where

$$
\begin{aligned}
& A_{1,2}^{\alpha}(\mathbf{q})=\frac{P_{q}^{\alpha}\left(\hbar \omega=\varepsilon_{1,2}(\mathbf{q})\right)}{4 Q_{q} \varepsilon_{1,2}(\mathbf{q})}, \\
& A_{3,4}^{\alpha}(\mathbf{q})=-\frac{P_{q}^{\alpha}\left(\hbar \omega=\varepsilon_{3,4}(\mathbf{q})\right)}{4 Q_{q} \varepsilon_{3,4}(\mathbf{q})} .
\end{aligned}
$$

The summation over the wave vectors is carried out in accordance with the formula

$$
\begin{aligned}
& \frac{1}{N} \sum_{\mathbf{q}} \Phi\left(|J(\mathbf{q})|^{2}\right)=\frac{1}{N} \sum_{\mathbf{q}} \Phi\left(t^{2}\left|\gamma_{q}\right|^{2}\right)= \\
& =\int d x \rho_{0}(x) \Phi\left(t^{2} x\right),
\end{aligned}
$$

$$
\begin{aligned}
& \left|\gamma_{\mathbf{q}}\right|^{2}=1+4 \cos \left(q_{x} \frac{a \sqrt{3}}{2}\right) \cos \left(q_{y} \frac{3}{2} a\right)+ \\
& +4 \cos ^{2}\left(q_{x} \frac{q \sqrt{3}}{2}\right)
\end{aligned}
$$

and $\rho_{0}(x)=\frac{1}{N} \sum_{\mathbf{q}} \delta\left(x-\left|\gamma_{\mathbf{q}}\right|^{2}\right)$ is an auxiliary function that characterizes the distribution over the squared energy and is related to the band density of states $g(\mathcal{E})$ for the graphene lattice as follows:

$\rho_{0}(x)=\frac{1}{2 \sqrt{x}} g(\sqrt{x})$.

ISSN 2071-0194. Ukr. J. Phys. 2014. Vol. 59, No. 10
The function $g(\mathcal{E})$ looks like

$$
g(\mathcal{E})=\frac{2}{\pi^{2}} \frac{|\mathcal{E}|}{\sqrt{Z_{0}}} F\left(\frac{\pi}{2}, \sqrt{\frac{Z_{1}}{Z_{0}}}\right)
$$

where

$$
\begin{aligned}
& Z_{0}= \begin{cases}(1+|\mathcal{E}|)^{2}-\frac{1}{4}\left(|\mathcal{E}|^{2}-1\right)^{2}, & |\mathcal{E}| \leqslant 1, \\
4|\mathcal{E}|, & 1 \leqslant|\mathcal{E}| \leqslant 3,\end{cases} \\
& Z_{1}= \begin{cases}4|\mathcal{E}|, & |\mathcal{E}| \leqslant 1 \\
(1+|\mathcal{E}|)^{2}-\frac{1}{4}\left(|\mathcal{E}|^{2}-1\right)^{2}, & 1 \leqslant|\mathcal{E}| \leqslant 3,\end{cases}
\end{aligned}
$$

and $F(\pi / 2, y)$ is the complete elliptic integral of the first kind (see works $[17,18]$ ).

For the $\delta$-function in formula (18), the following expression is used:

$$
\begin{aligned}
& \delta\left(\omega-\frac{\varepsilon_{i}(x)}{\hbar}\right)= \\
& =\delta\left(x-\tilde{x}_{i}\right) 2 \hbar\left|\varepsilon_{i}\right|\left|b+\frac{d}{2\left(\hbar^{2} \omega^{2}-a-b \tilde{x}_{i}\right)}\right|^{-1},
\end{aligned}
$$

where $\varepsilon_{i}(x)=\left.\varepsilon_{i}(\mathbf{q})\right|_{\left|\gamma_{q}\right|^{2} \rightarrow x}$, and $\tilde{x}_{i}$ is the root of the equation $\hbar \omega=\varepsilon_{i}(x)$. We also introduced the nota- 

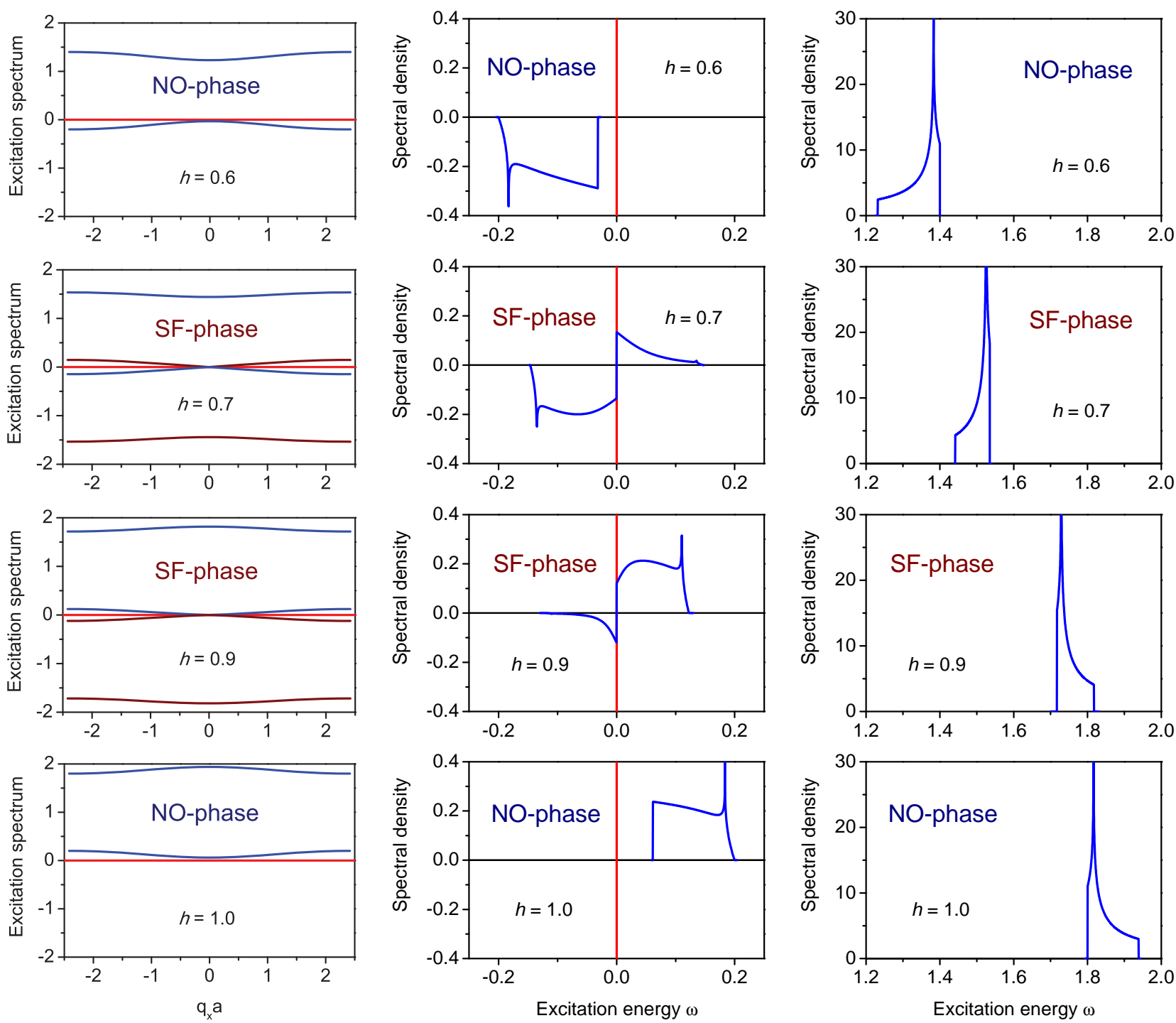

Fig. 5. Spectrum (left panels) and spectral density $\rho_{A}(\omega)$ (central and right panels) in the NO and SF phases at various energies $h$. The other parameters are $J(0)=1, \delta=0.8$, and $\Theta=0.05$. The plots of $\rho_{A}(\omega)$ for the lowest branch in the SF phase are not shown

tions

$$
\begin{aligned}
& a=\frac{E_{A}^{2}+E_{B}^{2}}{2}, \\
& b=\left\langle\sigma_{A}^{z}\right\rangle\left\langle\sigma_{B}^{z}\right\rangle \cos \vartheta_{A} \cos \vartheta_{B} t^{2} \equiv m \cos \vartheta_{A} \cos \vartheta_{B}, \\
& d=m\left(E_{A}+E_{B} \cos \vartheta_{A} \cos \vartheta_{B}\right) \times \\
& \times\left(E_{B}+E_{A} \cos \vartheta_{A} \cos \vartheta_{B}\right) .
\end{aligned}
$$

As a result, we obtain the following expression for the spectral density that characterizes the distribu- tion over the energy:

$$
\begin{aligned}
& \rho_{A}(\hbar \omega)=\sum_{i=1}^{4} \rho_{0}\left(\tilde{x}_{i}\right)\left\langle\sigma_{A}^{z}\right\rangle \times \\
& \times \frac{k+l \tilde{x}_{i}}{\left|2 b\left(\hbar^{2} \omega^{2}-a-b \tilde{x}_{i}\right)+d\right|}(-1)^{i-1}, \\
& k=\left[E_{A}\left(\cos ^{2} \vartheta_{A}+1\right)+2 \hbar \omega \cos \vartheta_{A}\right]\left(\hbar^{2} \omega^{2}-E_{B}^{2}\right), \\
& l=m \cos ^{2} \vartheta_{A}\left[\left(1+\cos ^{2} \vartheta_{B}\right) E_{B}-2 \hbar \omega \cos \vartheta_{B}\right] .
\end{aligned}
$$

An expression for $\rho_{B}(\hbar \omega)$ can be obtained from formulas (25) and (26) by swopping the indices: $A \rightleftarrows B$.

ISSN 2071-0194. Ukr. J. Phys. 2014. Vol. 59, No. 10 

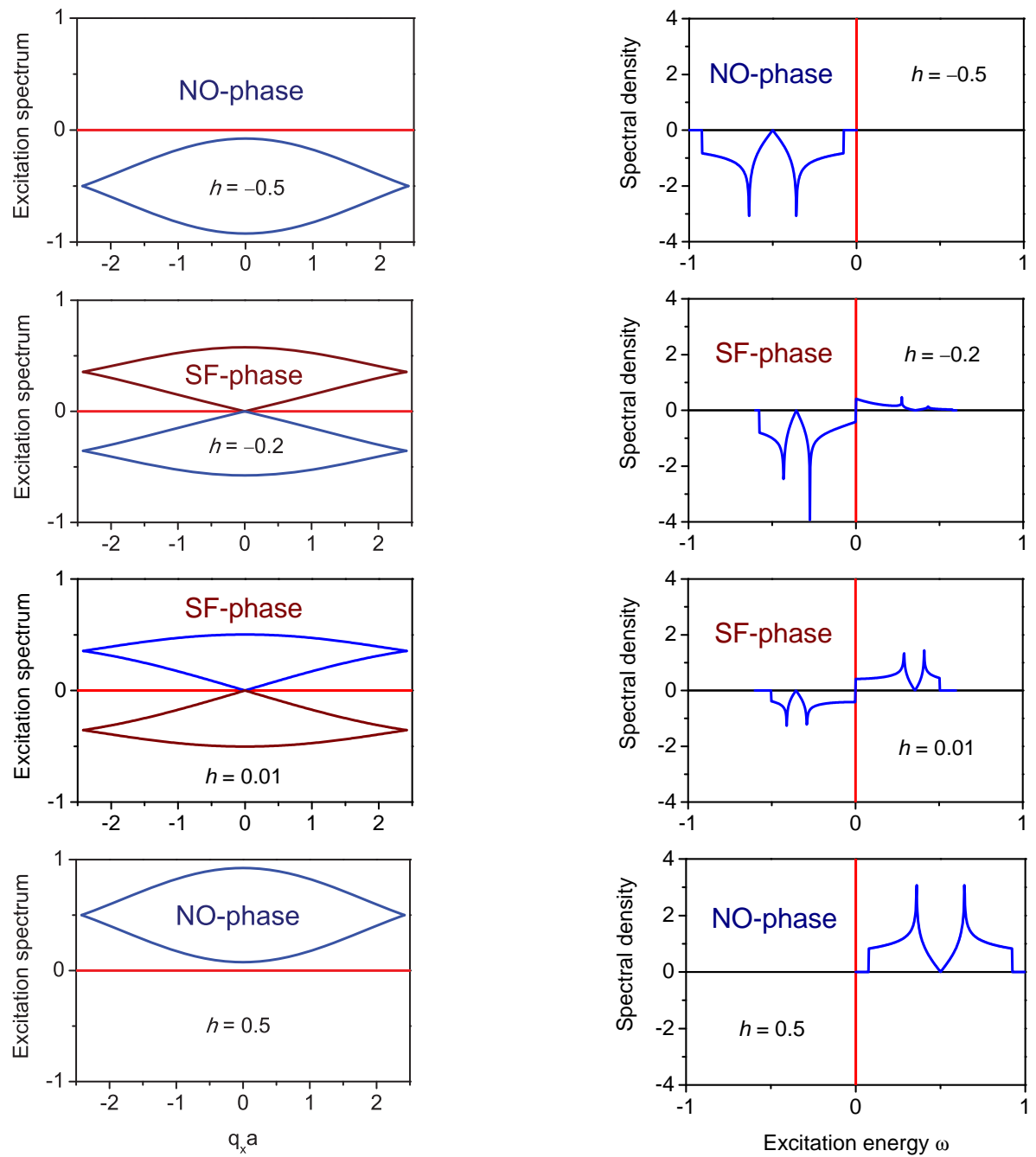

Fig. 6. Spectrum (left panels) and spectral density $\rho_{A}(\omega)$ (right panels) in the NO and SF phases at $\delta=0$ at various energies $h$. The other parameters are $J(0)=1$ and $\Theta=0.2$

Formula (25) was used in the numerical calculations of the curves $\rho_{A}(\hbar \omega)$ describing the frequency dependence of the spectral density for sublattice $A$. In Figs. 4 to 6 , the results obtained for various values of parameters $h$ and $\delta$ and various temperatures are shown. As was done earlier, the energetic quantities are reckoned in the $J(0)$-units.

A common feature of those plots is the sign change by the function $\rho_{A}(\omega)$ when crossing the point $\omega=0$. At $\omega<0$ (below the chemical potential level), the spectral density is negative, and, at $\omega>0$ (above the $\mu$-level), it is positive. The additional subbands that arise in the SF phase possess a much smaller weight at first, and their spectral densities can differ from the
$\rho_{A}$-values for the subbands in the $\mathrm{NO}$ phase even by several orders of magnitude (see Fig. 4). As the chemical potential goes deeper into the subband, where it is located (this process corresponds to the variation of the parameter $h$ (see Fig. 5)), the intensity redistribution between the positive and negative branches of the function $\rho_{A}$ takes place. The dispersion curves $\varepsilon_{i}(\mathbf{q})$ change insignificantly at that.

Figure 6 illustrates the reconstruction of the spectral density function $\rho_{A}(\hbar \omega)$, when the chemical potential moves within the band in the case where $\delta=0$. In this case, the band is not split (the gap is absent), and there are Dirac points in the spectrum. In the SF phase, their number doubles in comparison 
with the normal one. This fact manifests itself in the appearance of additional intermediate points in the spectral density $\rho_{A}$, where $\rho_{A}(\hbar \omega)=0$ (in a vicinity of those points, the frequency dependence of $\rho_{A}$ can be approximated by a linear function).

\section{Conclusions}

Our calculations of the dispersion laws $\varepsilon_{i}(\mathbf{q})$ and the spectral densities $\rho_{\alpha}(\hbar \omega)$ reveal main features in the band spectrum structure of hard-core bosons in a twodimensional lattice of the graphene type. The changes in their spectral characteristics at the transition from the NO to SF phase are described. It is shown that the form of the functions $\rho_{\alpha}(\hbar \omega)(\alpha=A, B)$ is much more sensitive to the values of energetic parameters of the system, including the position of the chemical potential of bosons, than the dispersion laws $\varepsilon_{i}(\mathbf{q})$ in the bosonic bands. For this reason, the functions $\rho_{\alpha}(\hbar \omega)$ can be considered as the basic characteristics of the band spectrum. The form of the dependences $\varepsilon_{i}(\mathbf{q})$ alone does not provide its exhaustive description.

It is found that, in the case of a lattice with energetically equivalent sublattices (at $\delta=0$ ), the Dirac points in the spectrum survive at the transition to the SF phase, and their number doubles. In the general case, the chemical potential of bosons remains outside a vicinity of the Dirac points, and it cannot be imposed onto them.

The results obtained can form a basis for the further researches of thermodynamic properties of the system of Bose particles in a honeycomb lattice with the graphene-type structure.

1. I.V. Stasyuk, I.R. Dulepa, and O.V. Velychko, Ukr. J. Phys. 59, 888 (2014).

2. M. Greiner, O. Mandel, T. Esslinger, T.W. Hänsch, and I. Bloch, Nature 415, 39 (2002).

3. M. Greiner, O. Mandel, T.W. Hänsch, and I. Bloch, Nature 419, 51 (2002).

4. P. Soltau-Panahi, J. Struck, A. Bick, W. Plenkers, G. Meineke, C. Becker, P. Windpassinger, K. Sengstock, P. Hauke, and M. Lewenstein, Nature Physics 7, 434 (2011).
5. D.-S. Lühmann, Phys. Rev. A 87, 043619 (2013).

6. Q.-Q. Lu and J.-M. Hou, Commun. Theor. Phys. 53, 861 (2010).

7. P. Soltau-Panahi, D.-S. Lühmann, J. Struck, P. Windpassinger, and K. Sengstock, Nature Physics 8, 71 (2012).

8. E. Albus, X. Fernandez-Gonzalvo, J. Mur-Petit, J.J. Garcia-Ripoli, and J.K. Pachos, Ann. Phys. 328, 64 (2013).

9. M.P.A. Fisher, P.B. Weichman, G. Grinstein, and D.S. Fisher, Phys. Rev. B 40, 546 (1989).

10. D. Jaksch, C. Bruder, J.I. Cirac, C.W. Gardiner, and P. Zoller, Phys. Rev. Lett. 81, 3108 (1998).

11. Z. Chen and B. Wu, Phys. Rev. Lett. 107, 065301 (2011).

12. S. Koghee, L.-K. Lim, M.O. Goerbig, and C. Morais-Smith, Phys. Rev. A 85, 023637 (2012).

13. R.T. Whitlock and P.R. Zilsel, Phys. Rev. 131, 2409 (1963).

14. I.V. Stasyuk and O. Vorobyov, Condens. Matter Phys. 16, 23005 (2013).

15. A.H. Castro Neto, F. Guinea, N.M.R. Peres, K.S. Novoselov, and A.K. Geim, Rev. Mod. Phys. 81, 109 (2009).

16. N.N. Bogolyubov, Izv. Akad. Nauk SSSR Ser. Fiz. 11, 77 (1947).

17. H.B. Rosenstock, J. Chem. Phys. 21, 2064 (1953).

18. J.P. Hobson and W.A. Nierenberg, Phys. Rev. 89, 662 (1953).

Received 10.06.14

Translated from Ukrainian by O.I. Voitenko

I.В. Стасюк, О.В. Величко, І.Р. Дулепа

ДОСЛІДЖЕННЯ БОЗОННОГО

СПЕКТРА ДВОВИМІРНИХ ОПТИЧНИХ

ІРАТОК ЗІ СТРУКТУРОЮ ТИПУ

ГРАФЕНУ. НАДПЛИННА ФАЗА

Р е $з$ ю м е

Досліджено енергетичний спектр системи бозе-атомів у надплинній фазі в оптичних гратках типу графену. Розрахунок законів дисперсії у зонах та одночастинкових спектральних густин проведено у наближенні хаотичних фаз у рамках формалізму жорстких бозонів. Описано їх зміни при переході від нормальної до надплинної фази. Під час такої перебудови збільшується вдвічі число підзон. У випадку енергетичної еквівалентності підграток діраківські точки у спектрі зберігаються, а їх кількість подвоюється. При енергетичній відмінності між підгратками точки Дірака відсутні. Показано, що форма спектральних густин чутлива до зміни температури та розташування хімічного потенціалу. 